\title{
Residential electrical vehicle charging strategies: the good, the bad and the ugly
}

\author{
Mingming LIU ( $\triangle$ ), Paul MCNAMARA, \\ Robert SHORTEN, Seán MCLOONE
}

\begin{abstract}
In recent years, a wide variety of centralised and decentralised algorithms have been proposed for residential charging of electric vehicles (EVs). In this paper, we present a mathematical framework which casts the EV charging scenarios addressed by these algorithms as optimisation problems having either temporal or instantaneous optimisation objectives with respect to the different actors in the power system. Using this framework and a realistic distribution network simulation testbed, we provide a comparative evaluation of a range of different residential EV charging strategies, highlighting in each case positive and negative characteristics.
\end{abstract}

Keywords Electric vehicle, Smart grid, Decentralised control, Centralised control, Demand side management

\section{Introduction}

Electric vehicles (EVs) avoid the use of petroleum, and so are seen as an efficient and effective replacement for traditional internal combustion engine based vehicles (ICEVs). They are expected to play a vital role worldwide in the near future in terms of addressing $\mathrm{CO}_{2}$ reduction

CrossCheck Date: 3 March 2015

Received: 1 October 2014 / Accepted: 14 January 2015/Published online: 13 May 2015

(C) The Author(s) 2015. This article is published with open access at Springerlink.com

M. LIU, Hamilton Institute, Maynooth University, Maynooth, Co., Kildare, Ireland

(凹) e-mail: mliu@eeng.nuim.ie

P. MCNAMARA, Department of Electronic Engineering,

Maynooth University, Maynooth, Co., Kildare, Ireland

R. SHORTEN, IBM Research, Dublin, Ireland

S. MCLOONE, School of Electronics, Electrical Engineering and Computer Science, Queen's University Belfast, Belfast, UK targets, combating climate change and improving energy security [1-3]. However, as EV penetration increases, the extra demand due to EV charging will have a considerable impact on the design and operation of electrical power systems [4]. In [5-10], it has been shown that the widespread adoption of EVs could negatively impact the distribution network if charging is not properly coordinated. Grid impacts of uncoordinated charging include, but are not limited to, increased voltage imbalance [7], increased grid losses [8], overloading [6], fluctuation of grid frequencies [11], and increased harmonic distortion [12]. In turn these effects result in a decrease in the operational efficiency of the grid and in the life span of electrical devices. It is also observed in these studies that in order to accommodate the extra EV charging loads, it will be necessary for utilities to invest in and reinforce grid infrastructures in heavily loaded areas, to accommodate both EV and household loads.

It has been shown in $[4,10]$ that by using suitable charging strategies, it is possible to mitigate some of the adverse impacts of charging, which could in turn reduce or postpone the need for infrastructure reinforcement. To date, there have been several different strategies proposed for charging groups of EVs connected to low-voltage distribution networks $[9,13-15]$. These strategies can be classified from the perspective of the different actors in the power system, which consist of EV consumers, distribution system operators (DSOs) and transmission system operators (TSOs) [16].

Consumer oriented algorithms typically focus on maximising the amount of charge that can be allocated to a customer in a given time period. It is usually desired to achieve this in a fair manner, providing a satisfactory quality of service (QoS), without violating system constraints, and minimising the cost to the customer. Many algorithms have been proposed using a centralised 
framework, the aim of which is to maximise the amount of charge allocated to customers [13, 17]. Here centralised implies that all the information in the network is available to a centralised controller, which in turn processes the information and decides the charge each $\mathrm{EV}$ will receive. These algorithms are typically based on linear programming techniques. While centralised coordination gives the best performance possible [18], centralised algorithms require access to global system information, which might not always be accessible, and centralised algorithms typically do not scale well [18-20]. For these reasons, several decentralised strategies have been proposed recently for EV charging [21-26]. In decentralised charging strategies individual EVs are given a certain level of decision making autonomy. Often individual EV chargers send a limited amount of information to a centralised unit which in turn provides some global coordination of their decisions, to a degree determined by the algorithm in use.

From the perspective of DSOs, charging strategies are usually designed to achieve a grid related objective such as the minimisation of power losses, while satisfying grid constraints, and providing satisfactory customer service. Several centralised coordination strategies of this nature have been developed in [15, 27-31]. Vehicle-to-grid (V2G) techniques for grid regulation have also been proposed. For example, in [28], V2G was used for grid regulation on a daily basis and for peak reduction at times of high demand. Some decentralised optimisation approaches have also been proposed to enhance grid regulation [32, 33].

Charging strategies aligned to TSO priorities include those focused on the scheduling of power supplies in an economic way, and those seeking to maximise the use of renewables on the grid. Centralised TSO based charging strategies include [34], where serial quadratic programming techniques were used to minimise the variance in the U.K. national demand profile, and [35] which examined the use of quadratic programming for load flattening under different penetrations of EVs. A more general study of adapting different centralised optimisation strategies for the coordination of EVs from the perspective of TSOs is presented in [36]. Several decentralised approaches developed from the TSO perspective are given in $[37,38]$. In [39-42], EVs are used for storage and control in order to maximise the utilisation of renewable energy. In [41], mixed-integer-linear-programming (MILP) was used to schedule EV charging loads in order to reduce charging costs and carbon emissions. In addition to this, EVs have been used to provide ancillary services, such as frequency control [43, 44].

With such a wide variety of algorithms available for EV charging it is desirable to have a common framework under which their performance can be compared. The objective of this paper is to present such a framework for residential charging of EVs, and to provide a comparative evaluation of a range of residential EV charging strategies within this framework using a realistic distribution network simulation testbed and representative charging scenarios. Through this comparison the positive and negative characteristics of each approach are identified, and the good, the bad and the ugly, so to speak, are highlighted.

The remainder of the paper is organised as follows. The proposed framework is introduced in Section 2. The EV charging algorithms considered are summarised in Section 3 and the testbed and simulation results are presented in Section 4. The results are discussed in Section 5 and finally conclusions are presented in Section 6.

\section{EV charging problem formulation}

A scenario in which a number of houses incorporating EVs are connected to a power distribution network is shown in Fig. 1. In this network, $S$ is defined as the number of distribution transformers. Let $\underline{S}$ denote the set $\{0,1, \ldots$, $S$. These transformers are then connected to the mediumvoltage (MV) substation bus, SubBus. The substation bus is powered by a transformer called $\mathrm{TR}(0)$, which connects to an external bulk power system.

A number of simplifications are used in the system model. The load power consumption in the network is discretised into $M$ discrete time slots, each of length $\Delta T$. For indexing purposes, let $\underline{M}$ denote the set $\{1,2, \ldots$, $M$. The loads are classified as non-EV loads, and EV loads, in the low-voltage (LV) areas. The number of houses across all LV areas is given by $N$. Let $\underline{N}$ denote the set $\{1$, $2, \ldots, N\}$. The index set of all houses connected to the transformer $T R(i)$ is given by $\phi_{h}^{i}$, and similarly the index

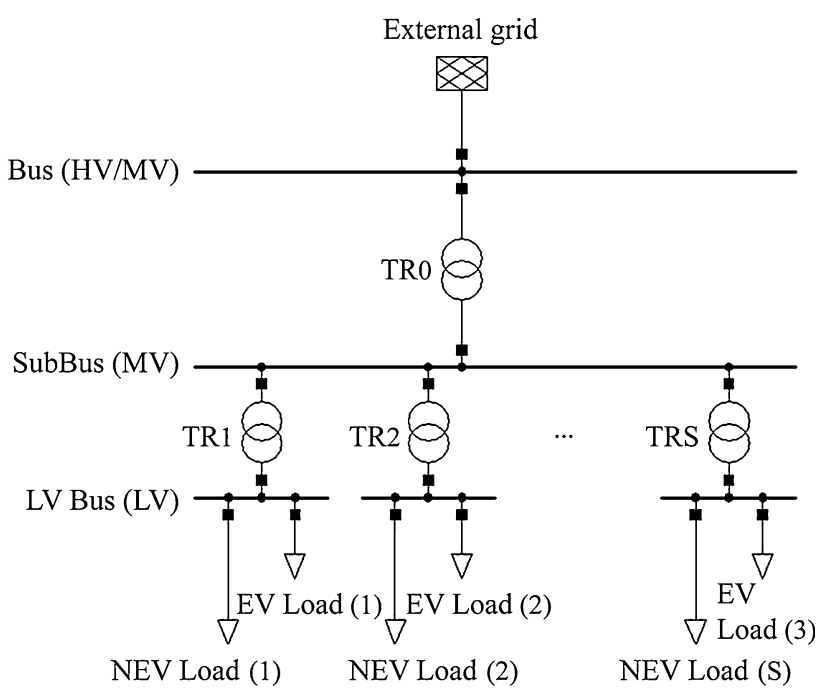

Fig. 1 Schematic diagram of the distribution network 
set of all EVs connected to the transformer $T R(i)$ is given by $\phi_{c}^{i}$.

The non-EV power consumption for the $j^{\text {th }}$ house at time slot $k$ is given by $h_{j}(k)$, and $c_{i}(k)$ denotes the charge rate of the $i^{\text {th }}$ active EV charge point at time slot $k$, for all $k \in \underline{M}$. The electricity price signal at sample time $k$, denoted $E(k)$, can represent either time-of-use (TOU) [45] or real-time pricing. The corresponding electricity price signal vector is given by $\boldsymbol{E}^{\mathrm{T}}:=[E(1), E(2), \ldots, E(M)]$. The charge rate vector for all EVs is given by $\mathbf{c}(k)^{\mathrm{T}}:=\left[c_{1}(k), c_{2}(k)\right.$, $\left.\ldots, c_{N}(k)\right]$ for all $k \in \underline{M}$. The charge rate profile for the $i^{\text {th }}$ $\mathrm{EV}$ is specified by $\mathbf{c}_{i}^{T}:=\left[c_{i}(1), c_{i}(2), \ldots, c_{i}(M)\right]$. A charge rate matrix is also defined as $C:=[\mathbf{c}(1), \mathbf{c}(2), \ldots, \mathbf{c}(M)]$. The plug-in time and plug-out time of the $i^{\text {th }} \mathrm{EV}$ are given by $\tau_{\text {in }}^{i}$ and $\tau_{\text {out }}^{i}$, respectively. Therefore, the $i^{\text {th }}$ EV must be charged within $\left[\tau_{\text {in }}^{i}, \tau_{\text {out }}^{i}\right]$. Let $P_{\text {av }}(k)$ denote the maximum available power that can be drawn from the external grid at time $k$ Due to the battery specification, each EV may have a different battery size $(\mathrm{kWh})$, and this parameter is denoted as $B_{\mathrm{i}}$ for the $i^{\text {th }}$ vehicle. The state-of-charge (SOC) for the $i^{\text {th }} \mathrm{EV}$ at time $k, \operatorname{SOC}_{i}(k)$, within $\left[\tau_{\mathrm{in}}^{i}, \tau_{\mathrm{out}}^{i}\right]$ is given by:

$\operatorname{SOC}_{i}(k)=\operatorname{SOC}_{i}\left(\tau_{\mathrm{in}}^{i}\right)+\sum_{i=\tau_{\mathrm{in}}^{i}}^{k-1} \frac{c_{i}(j) \cdot \Delta T}{B_{i}}$

Here $\operatorname{SOC}_{i}\left(\tau_{\text {in }}^{i}\right)$ is the initial SOC for the $i^{\text {th }} \mathrm{EV}$ when it plugs in. The maximum achievable SOC for the $i^{\text {th }} \mathrm{EV}$ is given by:

$S O C_{\max }^{i}=\min \left(1, \operatorname{SOC}_{i}\left(\tau_{\mathrm{in}}^{i}\right)+\frac{c_{\text {max }}^{i}\left(\tau_{\mathrm{out}}^{i}-\tau_{\mathrm{in}}^{i}\right) \cdot \Delta T}{B_{i}}\right)$

where $c_{\text {max }}^{i}$ is the maximum charge rate $(\mathrm{kW})$ of the $i^{\text {th }} \mathrm{EV}$ charge point. A feasible charging profile is a charging profile which satisfies both plug-in constraints and the state of charge condition, i.e. $\operatorname{SOC}_{i}(M)=\operatorname{SOC}_{\max }^{i}$ [46].

The following are also defined:

1) Aggregate non-EV base load at time $k$ is given by:

$b(k):=P_{\text {sub }}^{0}(k)-\sum_{i=1}^{N} c_{i}(k)$

2) Aggregate non-EV base load profile is defined as:

$\boldsymbol{b}^{\mathrm{T}}:=[b(1), b(2), \ldots, b(M)]$

\subsection{Plug-in constraints}

The ratings of both the EV charge point and vehicle battery impose constraints on the feasible charge rates which can be drawn from the charging socket by each EV. These are described in the following. For the $i^{\text {th }} \mathrm{EV}$, the maximum charge rate is denoted by $c_{\max }^{i}$. Considering the charging rate over the course of the full $M$ time slots and noting that charging can only take place when the EV is plugged in and not already fully charged, the constraints are given as follows:

$$
\begin{aligned}
& \text { if } \tau_{\text {in }}^{i} \leq k \leq \tau_{\text {out }}^{i} \\
& 0 \leq c_{i}(k) \leq c_{\max }^{i}, \\
& \text { If } k \leq \tau_{\mathrm{in}}^{i}, k \geq \tau_{\mathrm{out}}^{i}, \text { or } \operatorname{SOC}_{i}(k)=100 \% \text {, } \\
& c_{i}(k)=0
\end{aligned}
$$

\subsection{Power system constraints}

Some of the charging scenarios considered in the paper explicitly take account of constraints on power system transformer loading levels and voltage profiles. The total loading conditions for each transformer $P_{\text {sub }}^{i}(k), i \in \underline{S}$ at each time slot $k$ is inspected, and the voltage for each connected node $v_{\mathrm{i}}(k)$ is used to evaluate the voltage level at each sample step. In this context, the power flow for the $i^{\text {th }}$ transformer can be expressed as:

$P_{\mathrm{sub}}^{i}(k)=\sum_{j \in \phi_{h}^{i}} h_{j}(k)+\sum_{j \in \phi_{c}^{i}} c_{j}(k), i \in\{1,2, \ldots, S\}$

Accordingly, the total power flow for the main substation is defined as:

$P_{\mathrm{sub}}^{0}(k)=\sum_{j=1}^{s} P_{\mathrm{sub}}^{j}(k)$

In practice, (7) and (8) would not be evaluated explicitly; rather the relevant power flows would be measured directly at the transformers and would therefore also include distribution system losses. Letting $T R_{\text {max }}^{i}$ denote the maximum power rating for the $i^{\text {th }}$ transformer, the power flow constraint can be expressed as:

$P_{\text {sub }}^{i}(k) \leq T R_{\max }^{i}, i \in \underline{S}$.

In a similar fashion, defining the minimum acceptable voltage level as $v_{\min }$, the voltage constraint is given by:

$v_{i}(k) \geq v_{\min }, i \in \underline{N}$.

While the voltage level can be measured at each charge point, in general for monitoring purposes it only needs to be checked at the end of each phase since this will be the point where voltage violations will occur first.

\subsection{Cost functions}

Having established the model parameters and constraints, cost functions need to be defined to reflect the desired EV charging behaviour. Typically, cost functions for EV charging can be divided into two groups. The first are temporally based cost functions $J(\boldsymbol{C})$ that evaluate actions taken over a period of time. The second are 
instantaneous cost functions $J(\boldsymbol{c}(k))$ that only consider the current sample step.

In the case of temporal optimisation, which is essentially a scheduling problem, the optimum solution requires a priori knowledge of all relevant parameters over the optimisation horizon, $k \in \underline{M}$, such that the full $\boldsymbol{C}$ matrix can be determined, leading to sub-optimal solutions due to the uncertainty surrounding the predicted loads. These problems are typically computationally challenging and do not scale well. Typical temporal optimisation objectives include:

1) Minimising the total charging costs for all EVs over the course of all time slots [47]:

$J(\boldsymbol{C})=\sum_{k=1}^{M} \sum_{i=1}^{N} c_{i}(k) \cdot \Delta T \cdot E(k)$

2) Minimising the total energy losses on power transmission lines during EV charging period [48],

$J(\boldsymbol{C})=\sum_{k=1}^{M} \sum_{i=1}^{N_{l}} I_{l, k}^{2} \cdot R_{l} \cdot \Delta T$

where $N_{l}$ is the number of lines in a given area, $I_{l, k}$ the current on line $l$ at time $k$, and $R_{l}$ the resistance for line $l$.

3) Minimising the load variance (thereby flattening the load profile): $[37,46]$

$J(\boldsymbol{C})=\sum_{k=1}^{M}\left(P_{\text {sub }}^{0}(k)-\overline{P_{\text {sub }}^{0}}\right)^{2}$

where $\overline{P_{\text {sub }}^{0}}$ is defined as the average power consumption measured at the main transformer over the course of $M$ time slots.

In each case the optimisation problem is defined as

$$
\min _{\boldsymbol{C}} J(\boldsymbol{C})
$$

subject to: $\left\{\begin{array}{c}\text { Plug-in constraints } \\ \left.S_{S O C_{i}(M)=S O C_{\text {max }}^{i}, \forall i \in \underline{N}}\right\}\end{array}\right.$

where the SOC constraint is necessary to avoid the trivial solution $\boldsymbol{C}=0$.

Instantaneous cost functions are defined in terms of the information available at the current sample step, and hence are not subject to the uncertainty associated with load prediction, PEV availability, etc. Also, they are typically more amenable to decentralised implementation than temporal methods. However, as these functions only cater for the conditions at the current sample step, they typically cannot accommodate global or long term objectives. Examples of instantaneous optimisation objectives include:

1) Charge rate based fairness [33]: Here the objective is to minimise the difference between the charge rates of each EV while distributing the available power among EVs, that is: $\min _{\boldsymbol{c}(k)} \sum_{i=1}^{N}\left[c_{i}(k)-\frac{1}{N} \sum_{i=1}^{N} c_{i}(k)\right]^{2}$
subject to: $\left\{\begin{array}{c}\sum_{i=1}^{N} c_{i}(k)=P_{\mathrm{av}}(k), \forall k \in \underline{M} \\ \text { Plug-in constraints } \\ \text { Power system constraints (optional) }\end{array}\right\}$

2) Price based fairness [21]: In this case, charging fairness is defined from the perspective of the charging cost. In this problem, we expect the allocated charge rate for each EV to be proportional to the amount of money they would like to pay. A parameter $\omega_{i}$ is introduced for this purpose to reflect the level of willingness to pay of the $i^{\text {th }} \mathrm{EV}$ customer. The resulting optimisation problem can be expressed as:

$$
\text { subject to: }\left\{\begin{array}{c}
\min _{\boldsymbol{c}(k)} \sum_{i=1}^{N}\left[\frac{c_{i}(k)}{\omega_{i}}-\frac{1}{N} \sum_{i=1}^{N} \omega_{i} \cdot c_{i}(k)\right]^{2} \\
\sum_{i=1}^{N} c_{i}(k)=P_{\mathrm{av}}(k), \forall k \in \underline{M} \\
\frac{c_{i}(k)}{c_{j}(k)}=\frac{\omega_{i}}{\omega_{j}}, \forall i \neq j \in \underline{N}, \forall k \in \underline{M} \\
\text { Plug-in constraints } \\
\text { Power system constraints (optional) }
\end{array}\right\}
$$

Comment: The algorithms proposed in [33] and [21] can be thought of as providing an approximate distributed solution to the optimisation problems defined in (15) and (16), respectively. The constraint $\sum_{i=1}^{N} c_{i}(k)=P_{\mathrm{av}}(k)$ in both (15) and (16) is considered to avoid trivial solutions. In addition, the power system constraint is not considered in [21, 33]. However, as a necessary option for the grid, we shall consider this constraint in the proposed enhanced algorithms given in Section 3.

3) Maximising available power utilisation [17]: Here, utility companies wish to maximise the power delivered to charging EVs. The mathematical formulation of this problem is given as follows:

$$
\max _{\boldsymbol{c}(k)} \sum_{i=1}^{N} c_{i}(k)
$$

subject to: $\left\{\begin{array}{c}\text { Plug-in constraints } \\ \text { Power system constraints }\end{array}\right\}$

This can be generalised to:

$\max _{\boldsymbol{c}(k)} \sum_{i=1}^{N} f_{i}\left(c_{i}(k)\right)$

where $f_{i}$ is defined as a utility function associated with the $i^{\text {th }} \mathrm{EV}$, and can be used to influence charging behaviour. For instance, in [17], the functions were chosen such that 
EVs with lower SOC were given higher priority for charging.

\section{Charging strategies}

In this section, we introduce a number of different competing charging strategies that have been proposed in the literature, which we will evaluate on a realistic distribution network simulation testbed in Section 4.

\subsection{Uncoordinated charging strategy}

Uncoordinated charging, also known as uncontrolled charging or dumb charging, is where each EV begins charging at the maximum rate once it is plugged in, and continues charging at this rate until fully charged. The negative consequences of this approach for grid operation have been highlighted in many studies, see for example $[15,17]$. In the worst case scenario, if all EVs start to charge during peak load hours, then peak power requirements will be increased significantly and local distribution networks will likely be overloaded. This charging strategy can be viewed as the solution to the following temporal optimisation problem:

$\max _{\boldsymbol{C}} \sum_{k=1}^{M} \sum_{i=1}^{N} \operatorname{SOC}_{i}(k)$

\subsection{Decentralised AIMD algorithms}

The additive increase multiplicative decrease (AIMD) algorithm was originally applied in the context of decentralised congestion control in communication networks [49]. Reference [33] proposed applying the AIMD algorithm to EV charging problems and demonstrated its effectiveness in a number of practical scenarios. Roughly speaking, the key characteristic of the AIMD algorithm is that it guarantees an equitable "average" distribution of the available power between active EV charge points if each charge point chooses the same parameters. The elegance of the approach is that it achieves this desirable property while requiring only a minimal communication infrastructure and limited computational power at each EV. In addition, the simple communication topology and minimal communication bandwidth make it a highly scalable and cost effective solution. Further details could be found in [33].

\subsection{Enhanced distributed AIMD algorithms}

It should be noted that in the basic decentralised AIMD algorithm proposed in [33], many practical power system constraints were not considered. As an extension, we introduced a number of enhancements to the basic decentralised AIMD in [50] so that power system constraints on voltage and loading are taken into account.

The AIMD charging strategy is inherently an instantaneous algorithm with no temporal visibility, hence it cannot take a longer term view in terms of determining EV charge rates. However, a simple heuristic modification can be introduced to the available power calculation that allows the temporal context to be taken into account in a meaningful way with negligible impact on overall system complexity. The heuristic is to modulate the available power signal $P_{\mathrm{av}}(k)$ with TOU pricing information $E(k)$ so that an artificial reduction in available power is created at times of high electricity prices, that is:

$P_{\mathrm{av}}^{*}(k)=P_{\mathrm{av}}(k)-\delta\left(E(k)-E_{\mathrm{min}}\right)$.

Here $\delta$ is a constant tuning parameter, $E_{\min }$ is the minimum TOU price during the day. Please refer to [50] for further details on this heuristic and the Enhanced AIMD (EnAIMD) implementation.

Comment: Other approaches to fair decentralised EV charging have also been proposed. In particular, [51] describe a decentralised methodology for achieving fair EV charging under transmission constraints. This is based on solving a centralised mixed-integer-nonlinear-program (MINLP) problem using decomposition techniques, where each EV determines its own charging schedule by iteratively solving a knapsack-type optimisation problem. However, this method has a large communication overhead arising from the need for transacting signals between the EVs and a central authority. More sophisticated algorithms are also possible using the AIMD based approach; for instance [52] develop a V2G implementation that provides reactive power compensation capabilities to the grid.

\subsection{Distributed price feedback}

In [21], Fan borrowed the concept of congestion pricing in internet traffic control and introduced a willingness to pay (WTP) parameter to model the preference of user demand. Based on these ideas, he then developed a novel distributed framework for demand response and verified the convergence and dynamic behaviour of his adaptive algorithm, namely distributed price feedback (DPF), by case studies. With this framework in place, a novel distributed EV charging method was proposed such that each EV user could adapt their charging rate according to their personal preferences, maximising their own benefits.

\subsection{Enhanced distributed price feedback}

As was the case with AIMD, the original DPF framework does not consider the impact of EV charging on grid 
parameters. To address this we introduced an enhanced DPF (EnDPF) implementation in [53] that includes additional functionality similar to that introduced in EnAIMD.

\subsection{Ideal centralised instantaneous charging}

Here, an ideal centralised instantaneous charging (ICIC) solution based on a hierarchical structure is introduced. At sample step $k$, all charging EVs are required to send their charging requests $c_{\max }^{i}$ to their local transformers. Each local transformer calculates the charge rate for each EV $i$ in area $j$, taking into account the current local capacity $T R_{\max }^{j}-P_{\text {sub }}^{j}(k), * \forall \imath \in \phi_{\psi}^{\varphi}$, and forwards the power requirements to the main substation. If the total amount of requested power exceeds the available power, the main substation $\operatorname{TR}(0)$ allocates the available power to each substation in proportion to the requested values. Each substation then updates their EV charge rates accordingly and broadcasts the information to the charge points. This strategy is feasible for solving the following optimisation problem:

$\min _{\boldsymbol{c}(k)} \sum_{i=1}^{N}\left[c_{i}(k)-\frac{1}{N} \sum_{i=1}^{N} c_{i}(k)\right]^{2}$
subject to: $\left\{\begin{array}{c}\text { Plug-in constraints } \\ \sum_{i=1}^{N} c_{i}(k)=P_{\mathrm{av}}(k), \forall k \in \underline{M} \\ P_{\text {sub }}^{i}(k) \leq T R_{\text {max }}^{i}, \forall i \in \underline{S}\end{array}\right\}$

Note that voltage constraints are not considered within this formulation for the sake of simplicity.

\subsection{Optimal decentralised valley-filling charging}

A novel decentralised temporal optimisation algorithm, namely optimal decentralised valley-filling (ODVF), was proposed in [46] to optimally schedule EV charging to perform valley filling through an iterative process. It has been shown that the charging profile for each EV can reach optimality within a few iterations and that the approach provides satisfactory performance and is robust to errors in users' specifications and outdated signals.

In order to minimise the load variance (valley-filling) by the ODVF method, the following is solved:

$$
\left.\begin{array}{c}
\min _{c} \sum_{k=1}^{M}\left[\sum_{i=1}^{N} c_{i}(k)+b(k)\right]^{2} \\
\text { Plug-in constraints } \\
P_{\text {sub }}^{0}(k) \leq T R_{\max }^{0} \\
S O C_{i}(M)=S O C_{\max }^{i}, \forall i \in \underline{N}
\end{array}\right\}
$$

where $b(k)$ denotes the $k^{\text {th }}$ element of base load profile $\boldsymbol{b}$. Details of the algorithm implementation could be referred in [46].

\subsection{Decentralised selfish charging strategy}

In this charging strategy, it is assumed that charging is conducted in a decentralised fashion by each EV guided only by TOU pricing information (assumed to be available a priori) and plug-in constraints. TOU information motivates EV owners to shift their demands to cheaper price periods. Therefore, in decentralised selfish charging (DSC), each EV optimises its charging schedule in order to meet its charging requirements at the minimum cost with no regard for the impact on the power system. Mathematically, the DSC formulation can be viewed as solving the follows optimisation problem:

$$
\begin{aligned}
\min _{c} \sum_{k=1}^{M} c_{i}(k) \cdot \Delta T \cdot E(k), \forall i \in \underline{N} \\
\text { subject to: } \quad\left\{\begin{array}{c}
\text { Plug-in constraints } \\
\operatorname{SOC}_{i}(M)=S O C_{\max }^{i}, \forall i \in \underline{N}
\end{array}\right\}
\end{aligned}
$$

\subsection{Centralised cost minimisation charging strategy}

In this section, a centralised charging cost minimisation (CCCM) strategy based on linear programming is proposed to minimise the total cost of charging EVs. Using this method, the charge rate matrix of all vehicles $\boldsymbol{C}$ is determined at a centralised control center. Rather than updating the charge rate locally according to some feedback signals (e.g. price signal) at every time slot, centralised approaches are more amendable to fulfilling temporal based objectives (e.g. minimising total charging costs, valley-filling). We assume that at the beginning of the scheduling window, all essential information is provided to the optimisation program for computation in the control center. This information includes the predicted base load $\boldsymbol{b}$ and the charging schedule of each EV, i.e., $\tau_{\text {in }}^{i}$ and $\tau_{\text {out }}^{i}$.

In this case, the optimisation problem is taken as a centralised scheduling problem to minimise total charging costs. The mathematical formulation can be defined considering the SOC of all EVs, power system constraints and plug-in constraints:

$$
\begin{gathered}
\min _{c} \sum_{k=1}^{M} \sum_{i=1}^{N} c_{i}(k) \cdot \Delta T \cdot E(k) \\
\text { subject to: }\left\{\begin{array}{c}
\text { Plug-in constraints } \\
\text { Power system constraints } \\
\operatorname{SOC}_{i}(M)=S O C_{\text {max }}^{i}, \forall i \in \underline{N}
\end{array}\right\}
\end{gathered}
$$

Comment: If the power system constraints are omitted, the solution to the optimisation problem defined in (24) is mathematically equivalent to the solution of (23). Thus, CCCM is an enhancement of DSC since it is not practical to incorporate power system constraints in the DSC method. However, this is at the expense of substantial communication and computation overhead. 


\subsection{Centralised load-variance-minimisation charging}

In this case, the optimisation problem is formulated as a quadratic programming problem, the aim of which is to flatten the overall load profile, i.e., valley-filling. Compared to the ODVF method, as defined in Section 3.7, this charging strategy, denoted CLVM, gathers all the necessary information from both the grid and EV customers before solving the quadratic optimisation problem, in order to determine the optimal charge rate matrix $\boldsymbol{C}$ before charging commences. Mathematically, the optimisation problem is given by (13) and (14) as presented in Section 2.3 .

\section{Case studies and results}

\subsection{Simulation set-up}

In order to compare the performance of the different charging strategies, a one day simulation was run with $\Delta T$ set to $5 \mathrm{~min}$, i.e. $M=288$. The simulation was conducted on a typical residential low voltage distribution network with $S=3$ and $N=160$. The houses are distributed evenly across phases with maximum $50 \% \mathrm{EV}$ penetration randomly connected in three household areas.

This distribution network was modelled and implemented using a custom OpenDSS/ Matlab simulation platform. OpenDSS [54], an open source electric power system distribution system simulator, was used to simulate the power system and calculate the instantaneous power flows and voltage profiles for the test network. Matlab was used to simulate typical residential EV connection, SOC and disconnection patterns (randomly generated for each $\mathrm{EV}$ ) and to create a wrapper programme to simulate the operation of the network over a period of time for varying household and EV loads based on various charging strategies. The topology of the network is given in Fig. 1.

The non-EV household load profiles for each scenario were generated based on residential customer smart meter electricity trial data provided by the Commission for Energy Regulation (CER) [55] in Ireland. The assumptions on EV travelling patterns, and hence SOC and plug-in/out probability distributions, were taken from [56]. For comparison purposes, the same plug-in/out and SOC values were used with each method considered. It was assumed that all EVs charged overnight and that once an EV was plugged in, it only physically plugged out at the scheduled plug-out time.

We now present a comparison of the listed charging strategies grouped according to their different characteristics as follows.
1) Uncoordinated charging strategy

2) Fairness based strategies: AIMD, DPF, EnAIMD, EnDPF and ICIC and their TOU price adjusted extensions

3) Cost minimisation strategies: DSC and CCCM

4) Valley-filling strategies: ODVF and CLVM

\subsection{Evaluation of the uncoordinated charging strategy}

As already noted in Section 3.1, uncontrolled charging is where each $\mathrm{EV}$ is charged at its maximum rate once it is plugged in and continues charging at this rate until it is fully charged. In this section, different penetration levels of EVs on the network were examined to evaluate the impact of uncontrolled charging on both power grid and customers. As shown in Fig. 2, the minimum non-EV voltage on all buses during the peak-periods was found to be 0.9538 p.u. (219.36 V). With uncontrolled EV charging coinciding with peak-power several bus voltages drop below 0.95 p.u. (the minimum accepted voltage on the grid), with the minimum voltage found to be $0.9151 \mathrm{p}$. u. around $7 \mathrm{pm}$.

The power flow at the substation and all local transformers are marginally overloaded during peak times as a result of EV charging in the case of $10 \% \mathrm{EV}$ penetration, as can be seen in Fig. 3. In the case of $50 \% \mathrm{EV}$ penetration, maximum loading occurs around $8 \mathrm{pm}$ and exceeds the available power by $43.56 \%$. Thus, for our test distribution network, uncontrolled charging at $50 \% \mathrm{EV}$ penetration cannot be supported.

\subsection{Evaluation of fairness based strategies}

In this section, the different fairness based charging strategies are discussed. In the DPF method, the WTP parameter was chosen to be 1, 2 or 3 randomly by each EV.

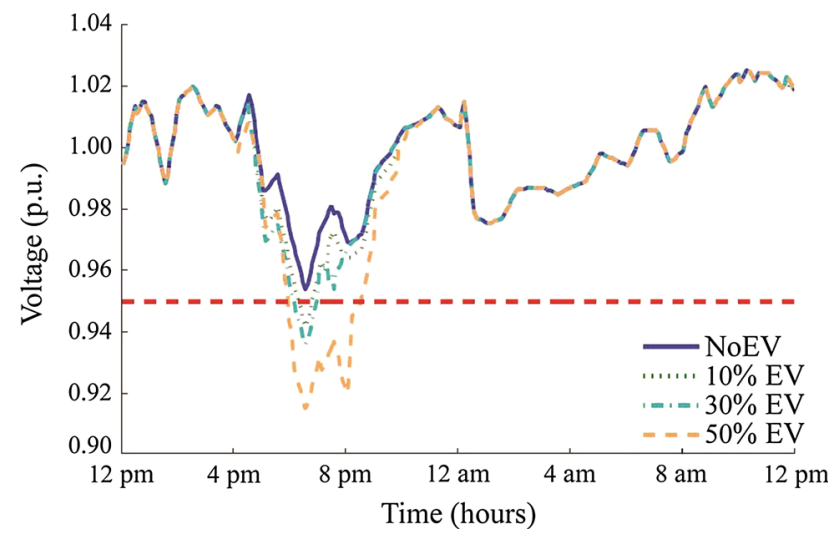

Fig. 2 Comparison of the minimum voltage profiles with different levels of EV penetration using uncontrolled charging 


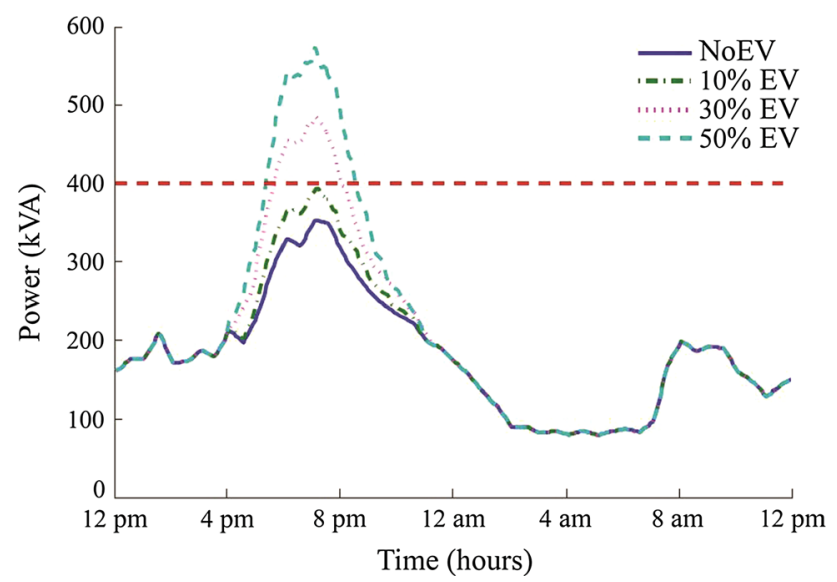

Fig. 3 Comparison of the power consumption at the main substation with different levels of EV penetration using uncontrolled charging

In the case of the ideal algorithm, results are presented only for the implementation without the voltage limitation condition. For all methods, the utility regulatory factor $\delta$ in (20) was set to 10 . The resultant power and voltage profiles, and the profile of the loading of the second transformer are presented in Figs. 4, 5, and 6, respectively.

The results show that both AIMD and DPF provide a good approximation to the ideal solution, and as such are competitive alternatives to the ideal solution given the substantially reduced communication overhead associated with their distributed implementation. DPF has the advantage that by using its price-feedback approach, it is able to provide users with a charge rate proportional to their WTP in their local area (local fairness is maintained at each given time instance), which is demonstrated in Fig. 7. The averaged charge rate obtained by DPF method for EVs connected in a given area (TR(2)) was calculated as $0.94,1.42$ and $1.76 \mathrm{~kW}$ by means of slow, normal and fast charging, respectively. It should be mentioned that the average charge rate for the different EVs is not necessarily proportional to

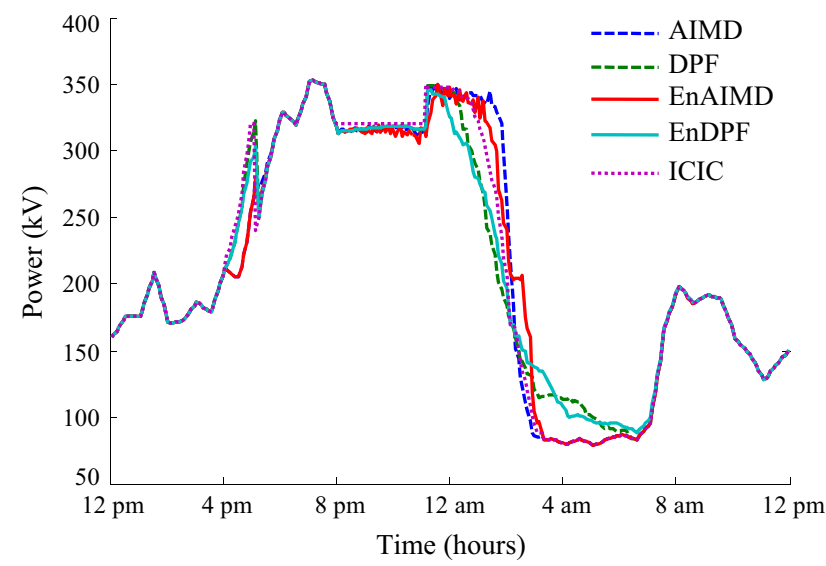

Fig. 4 Comparison of the power consumption at the main substation with different charging strategies

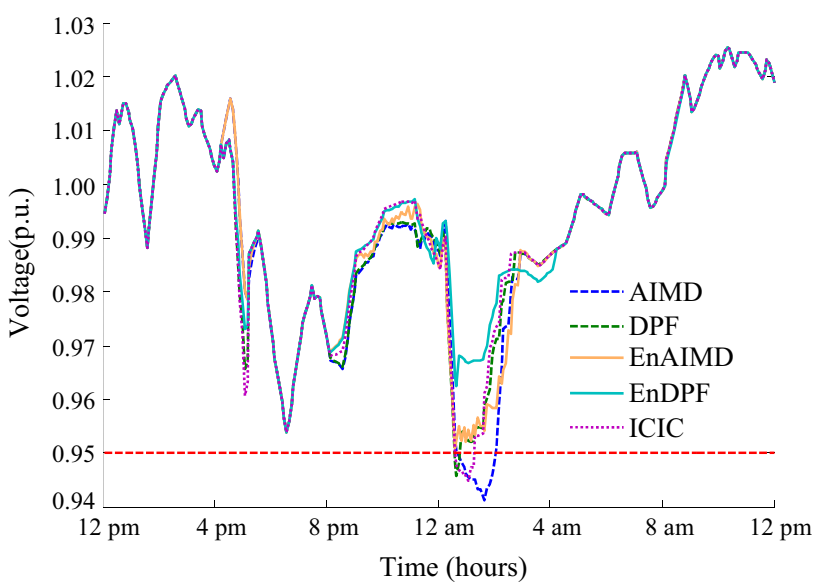

Fig. 5 Comparison of the minimum voltage profile with different charging strategies

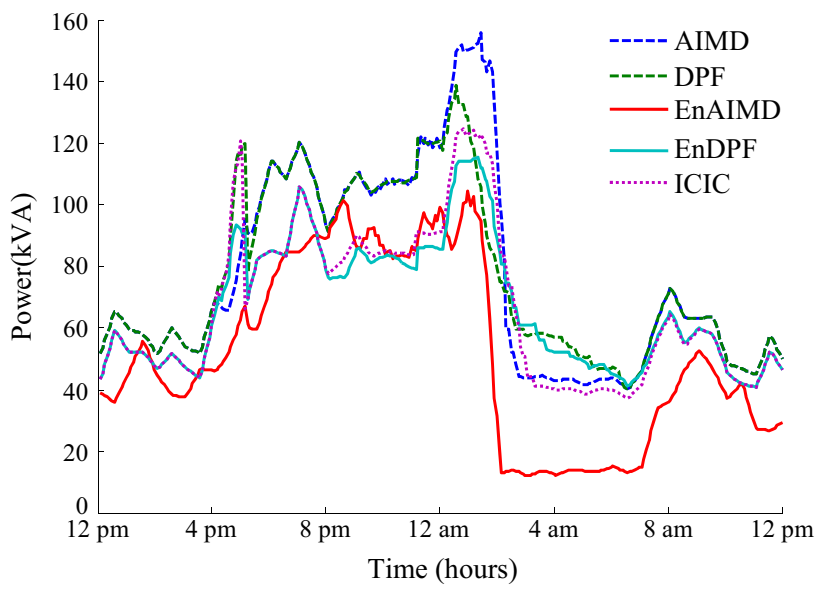

Fig. 6 Comparison of the loading conditions on transformer TR(2) with different charging strategies

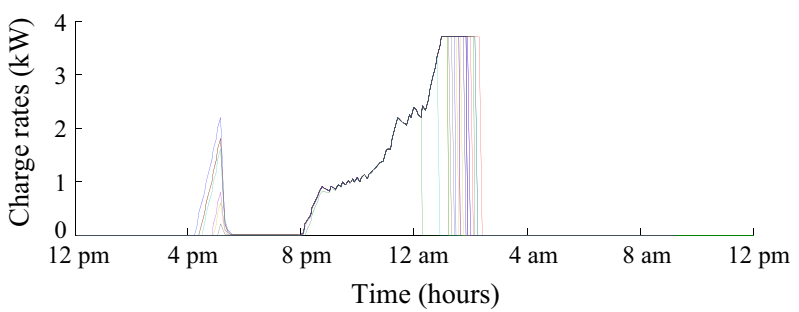

(a) Enhanced AIMD charging with price adjusted available power

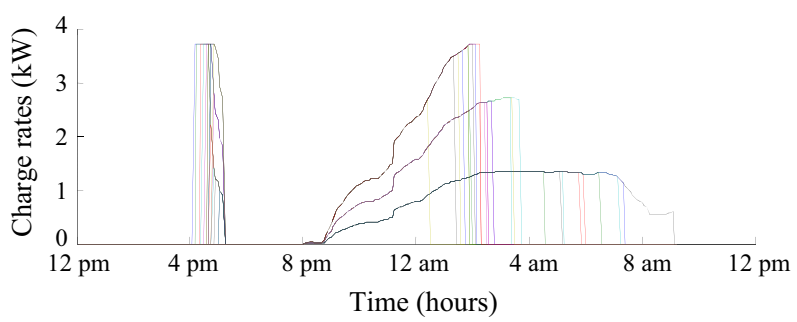

(b) Enhanced DPF charging with price adjusted available power

Fig. 7 Comparison of the charge rates in the same area (both connected to TR(2)) 
Table 1 A comparison of simulation results for various EV charging strategies (50\% EV penetration)

\begin{tabular}{|c|c|c|c|c|c|c|c|c|c|c|}
\hline Strategies & $\begin{array}{l}\text { Ave costs } \\
\text { (cents/kWh) }\end{array}$ & $\begin{array}{l}\text { Ave rate } \\
(\mathrm{kW})\end{array}$ & $\begin{array}{l}\text { Min volt } \\
\text { (p.u.) }\end{array}$ & $\begin{array}{l}\text { Max TR } \\
\text { loading }(\%)\end{array}$ & $\begin{array}{l}\text { Overload } \\
\text { duration (h) }\end{array}$ & $\begin{array}{l}\text { Ave } \\
\text { time (h) }\end{array}$ & $\begin{array}{l}\text { Std } \\
\text { time } \\
\text { (h) }\end{array}$ & $\begin{array}{l}\text { Min } \\
\text { time (h) }\end{array}$ & $\begin{array}{l}\text { Max } \\
\text { time (h) }\end{array}$ & $\begin{array}{l}\text { Overall } \\
\text { assessment }\end{array}$ \\
\hline No EV & $\mathrm{n} / \mathrm{a}$ & $\mathrm{n} / \mathrm{a}$ & 0.954 & 112.93 & 2.25 & $\mathrm{n} / \mathrm{a}$ & $\mathrm{n} / \mathrm{a}$ & $\mathrm{n} / \mathrm{a}$ & $\mathrm{n} / \mathrm{a}$ & $\mathrm{n} / \mathrm{a}$ \\
\hline Uncontrolled & 22.32 & 3.70 & 0.915 & 177.68 & 14.83 & 2.80 & 0.38 & 1.83 & 3.83 & $\mathrm{Bad}$ \\
\hline AIMD & 13.36 & 1.38 & 0.940 & 122.49 & 9.33 & 7.53 & 0.88 & 5.42 & 9.17 & $\mathrm{Bad}$ \\
\hline AIMD(P) & 11.21 & 1.25 & 0.941 & 113.92 & 6.58 & 8.34 & 1.05 & 5.92 & 10.08 & $\mathrm{Bad}$ \\
\hline EnAIMD & 12.74 & 1.36 & 0.953 & 112.93 & 2.75 & 7.89 & 1.63 & 4.58 & 10.83 & Good \\
\hline $\operatorname{EnAIMD(P)}$ & 11.18 & 1.23 & 0.950 & 112.93 & 2.25 & 8.56 & 1.29 & 5.83 & 11.00 & Good \\
\hline DPF & 13.93 & 1.36 & 0.946 & 122.17 & 9.33 & 7.86 & 1.84 & 4.42 & 11.42 & $\mathrm{Bad}$ \\
\hline $\mathrm{DPF}(\mathrm{P})$ & 11.30 & 1.22 & 0.947 & 113.91 & 5.83 & 8.71 & 1.81 & 5.00 & 12.33 & $\mathrm{Bad}$ \\
\hline EnDPF & 13.63 & 1.35 & 0.953 & 112.93 & 5.17 & 8.00 & 2.42 & 3.92 & 14.50 & Good \\
\hline $\operatorname{EnDPF}(\mathrm{P})$ & 11.22 & 1.16 & 0.954 & 112.93 & 2.33 & 9.19 & 2.02 & 5.83 & 14.58 & Good \\
\hline ICIC & 13.84 & 1.41 & 0.951 & 124.47 & 9.92 & 7.41 & 1.11 & 4.67 & 9.25 & Ugly \\
\hline $\mathrm{ICIC}(\mathrm{P})$ & 11.20 & 1.27 & 0.945 & 116.01 & 5.75 & 8.24 & 1.10 & 5.67 & 10.08 & Ugly \\
\hline ODVF & 10.46 & 1.36 & 0.958 & 112.93 & 2.25 & 7.31 & 0.74 & 5.92 & 8.58 & Good \\
\hline CLVM & 10.46 & 1.36 & 0.958 & 112.93 & 2.25 & 7.31 & 0.74 & 5.92 & 8.58 & Ugly \\
\hline DSC & 10.00 & 1.13 & 0.954 & 112.93 & 2.61 & 8.88 & 0.26 & 7.92 & 9.00 & Ugly \\
\hline ССCM & 10.00 & 1.11 & 0.954 & 112.93 & 2.25 & 8.89 & 0.27 & 7.92 & 9.00 & Ugly \\
\hline
\end{tabular}

their WTP parameters, due to the impact of local power system and charging infrastructure constraints.

Note that the results for the scenarios without priceadjusted available power are summarised in the Table 1.

\subsection{Evaluation of cost minimisation strategies}

In this section, the two charging strategies designed to minimise charging costs, namely DSC and CCCM, are applied to the distribution network with $50 \% \mathrm{EV}$ penetration and TOU pricing as given in Fig. 8. Simulation results show that DSC, which has no regard for power system constraints, violates local transformer loading constraints even if EV charging is delayed to off-peak times (around $12 \mathrm{am}$ ), while $50 \% \mathrm{EV}$ penetration can be comfortably accommodated on the network with CCCM, as shown in Fig. 9 and the transformer loading indexes reported in Table 1. However, CCCM needs to gather information from EVs and also from the power grid, hence it has substantial scalability issues compared to DSC.

\subsection{Evaluation of valley filling charging strategies}

For the following simulations, a simplification is made by assuming that all EVs share a common plug-out time of 6 am. As can be seen in Fig. 10 and Table 1, the performance of the ODVF method, when it is allowed a number of iterations, is comparable to that of CLVM. Most significantly, the ODVF method gives a significant reduction in computation time compared to the CLVM method as

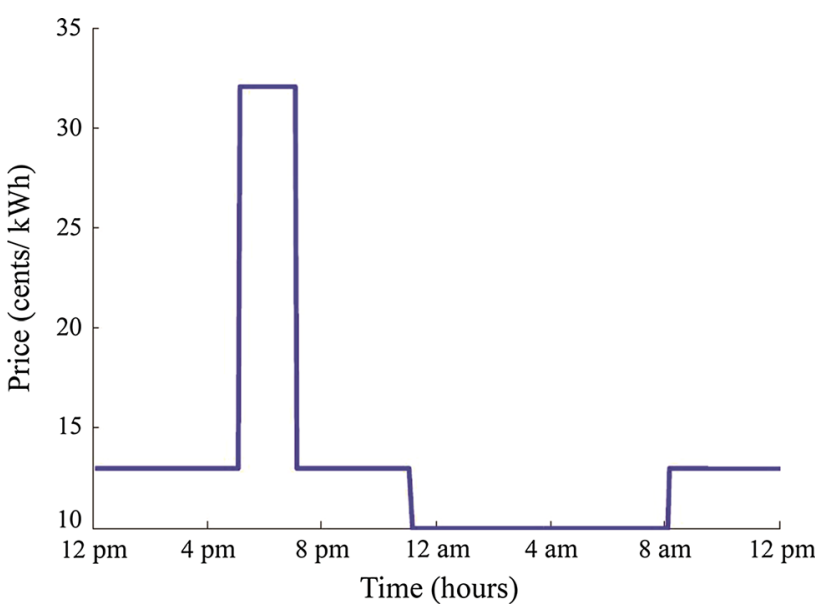

Fig. 8 Diagram of the TOU electricity price

illustrated in Tables 2 and 3. These times are calculated using quadprog in Matlab using a Dell computer on Win7 64bits operation system (RAM: 6GB, CPU: Inter(R) Core(TM) i7-2600, 3.4GHz). Thus it is easy to observe the significant advantages of applying the ODVF method versus the CLVM method from the perspective of computation times.

The performance of the ODVF method was also evaluated from the perspective of the mean square error (MSE) between the iterative load profile and the optimal aggregated load profile using the CLVM method. This showed that ODVF is able to converge to within $90 \%$ of the CLVM optimum in less than 6 iterations. 


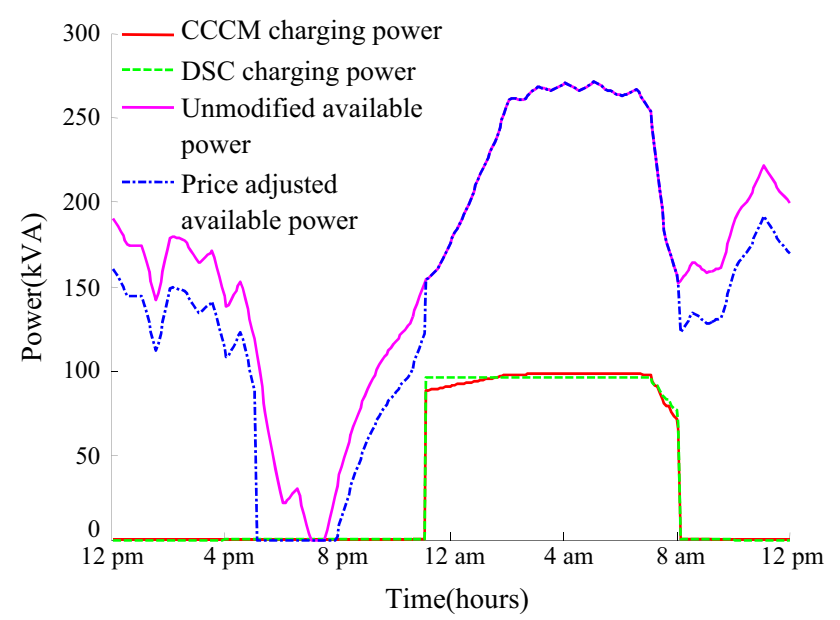

Fig. 9 Comparison of the EV load when using CCCM and DSC with the price adjusted available power modification

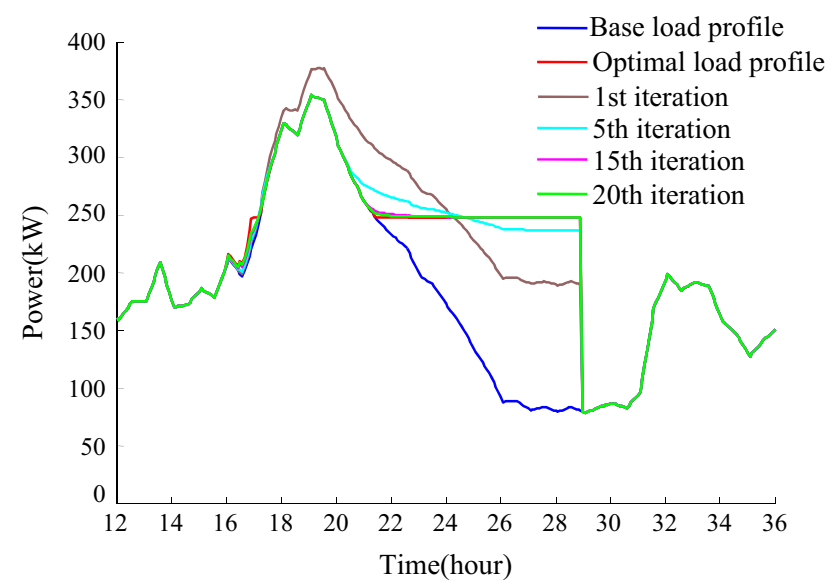

Fig. 10 Comparison of the optimal aggregated load profile obtained using CLVM and the iterative ODVF methods

\section{Discussion}

In general, uncontrolled charging provides the best performance in terms of its objective, i.e., minimising customer charging times. Similarly, by applying the basic AIMD and DPF based charging strategies, charging fairness can be achieved for each customer under appropriate assumptions, while the basic DPF method can adjust this fairness according to the WTP specified by individual customers.

However, typically these approaches violate grid constraints, as can be seen in Table 1 and Figs. 2 and 3. The addition of constraints allows the respective charging goals to be met while respecting grid constraints, as shown in Table 1 and Figs. 4, 5 and 6. While centralised algorithms achieve the best performance in terms of their stated goals, including satisfying power system constraints, they require global information and also do not scale well, as mentioned previously. For example, here, the CLVM method has the
Table 2 ODVF computation time for different iteration counts $(50 \%$ EV penetration)

\begin{tabular}{lc}
\hline ODVF & Time (s) \\
\hline 5 & 6.08 \\
10 & 11.41 \\
15 & 16.96 \\
20 & 22.79 \\
\hline
\end{tabular}

Table 3 CLVM computation times with different levels of EV penetration

\begin{tabular}{lr}
\hline CLVM $(\%)$ & Time (s) \\
\hline 10 & 20.87 \\
20 & 104.72 \\
30 & 236.02 \\
40 & 789.23 \\
50 & 2105.78 \\
\hline
\end{tabular}

longest simulation times. Decentralised algorithms, like the ODVF algorithm, approach the performance of centralised algorithms and are scalable. However, there is a large communication overhead associated with such algorithms.

Therefore, the modified AIMD and DPF algorithms show much promise. These algorithms provide a trade-off between a small communication overhead and almost optimal performance in terms of their grid objectives, and can be coordinated to approximately achieve some temporal objectives without the necessity of accurate load prediction, i.e. by applying a price-adjusted available power heuristic.

\section{Conclusions}

A mathematical framework for formulating EV charging problems has been presented that incorporates both power system and charging infrastructure constraints and caters for both instantaneous and temporal optimisation objectives. Within this framework, several charging strategies were evaluated. These algorithms were tested on a power system distribution level testbed using a hybrid Matlab/ OpenDSS platform, and using realistic demand and charging profiles. Of the algorithms considered, it was found that those algorithms that ignored system constraints typically violated them for large EV penetrations (BAD). Of the algorithms that considered power system constraints, the modified AIMD and DPF algorithms provided the best trade off in terms of achieving almost optimal performance in terms of their grid objectives while satisfying constraints and maintaining a small communication overhead (GOOD) in comparison to the other communication based optimal algorithms considered (UGLY). 
Acknowledgment The authors would like to thank the Irish Social Science Data Archive (ISSDA) for providing access to the CER Smart Metering Project data. The authors also gratefully acknowledge funding for this research provided by Science Foundation Ireland (Grant 11/PI/1177 and Grant 09/SRC/E1780).

Open Access This article is distributed under the terms of the Creative Commons Attribution 4.0 International License (http:// creativecommons.org/licenses/by/4.0/), which permits unrestricted use, distribution, and reproduction in any medium, provided you give appropriate credit to the original author(s) and the source, provide a link to the Creative Commons license, and indicate if changes were made.

\section{References}

[1] Huo H, Zhang Q, Michael Q et al (2010) Environmental implication of electric vehicles in China. Environ Sci Technol 44(13):4856-4861

[2] Singh M, Vyas A, Steiner E (2003) Vision model: description of model used to estimate the impact of highway vehicle technologies and fuels on energy use and carbon emissions to 2050. ANL/ESD/04-1, Argonne National Laboratory (ANL), Argonne

[3] Valentine-Urbschat M, Bernhart W (2009) Powertrain 2020The future drives electric. Roland Berger Strategy Consultants, Munich

[4] Lopes JAP, Soares FJ, Almeida PMR (2011) Integration of electric vehicles in the electric power system. Proc IEEE 99(1):168-183

[5] Gerkensmeyer C, Kintner-Meyer MCW, DeSteese JG (2010) Technical challenges of plug-in hybrid electric vehicles and impacts to the US power system: distribution system analysis. PNNL-19165, Pacific Northwest National Laboratory (PNNL), Richland

[6] Shao SN, Pipattanasomporn M, Rahman S (2009) Challenges of PHEV penetration to the residential distribution network. In: Proceedings of the IEEE power and energy society general meeting, Calgary, 26-30 July 2009, 8 pp

[7] Putrus GA, Suwanapingkarl P, Johnston D et al (2009) Impact of electric vehicles on power distribution networks. In: Proceedings of the IEEE vehicle power and propulsion conference (VPPC'09), Dearborn, 7-10 Sept 2009, pp 827-831

[8] Taylor J, Maitra A, Alexander M et al (2010) Evaluations of plug-in electric vehicle distribution system impacts. In: Proceedings of the IEEE power and energy society general meeting, Minneapolis, 25-29 Sept 2009, 6 pp

[9] Farkas C, Szabo KI, Prikler L (2011) Impact assessment of electric vehicle charging on a LV distribution system. In: Proceedings of the 3rd international youth conference on energetics (IYCE'11), Leiria, 7-9 July 2011, 8 pp

[10] Matrose C, Helmschrott T, Godde M et al (2012) Impact of different electric vehicle charging strategies onto required distribution grid reinforcement. In: Proceedings of the 2012 IEEE transportation electrification conference and expo (ITEC'12), Dearborn, 18-22, June 2012, 5 pp

[11] Takagi M, Yamaji K, Yamamoto H (2009) Power system stabilization by charging power management of plug-in hybrid electric vehicles with LFC signal. In: Proceedings of the IEEE vehicle power and propulsion conference (VPPC'09), Dearborn, 7-10 Sept 2009, pp 822-826

[12] Kutt L, Saarijarvi E, Lehtonen M et al (2013) A review of the harmonic and unbalance effects in electrical distribution networks due to EV charging. In: Proceedings of the 12th international conference on environment and electrical engineering (EEEIC'13), Wroclaw, 5-8 May 2013, pp 556-561

[13] Richardson P, Flynn D, Keane A (2012) Optimal charging of electric vehicles in low-voltage distribution systems. IEEE Trans Power Syst 27(1):268-279

[14] Clement K, Haesen E, Driesen J (2009) Coordinated charging of multiple plug-in hybrid electric vehicles in residential distribution grids. In: Proceedings of the IEEE/PES power systems conference and exposition (PSCE'09), Seattle, 15-18 Mar 2009, 7 pp

[15] Clement-Nyns K, Haesen E, Driesen J (2010) The impact of charging plug-in hybrid electric vehicles on a residential distribution grid. IEEE Trans Power Syst 25(1):371-380

[16] Verzijlbergh RA, Lukszo Z, Ilic MD (2012) Comparing different EV charging strategies in liberalized power systems. In: Proceedings of the 9th international conference on the European energy market (EEM), Florence, 10-12 May 2012, 8 pp

[17] Richardson P, Flynn D, Keane A (2012) Local versus centralized charging strategies for electric vehicles in low voltage distribution systems. IEEE Trans Smart Grid 3(2):1020-1028

[18] Kersting WH (2012) Distribution system modelling and analysis, 3rd edn. CRC Press, Boca Raton

[19] Negenborn RR, De Schutter B, Hellendoorn J (2008) Multiagent model predictive control for transportation networks: Serial versus parallel schemes. Eng Appl Artif Intel 21(3):353-366

[20] McDaniel P, McLaughlin S (2009) Security and privacy challenges in the smart grid. IEEE Secur Priv 7(3):75-77

[21] Fan Z (2012) A distributed demand response algorithm and its application to PHEV charging in smart grids. IEEE Trans Smart Grid 3(3):1280-1290

[22] Ramchurn SD, Vytelingum P, Rogers A et al (2011) Agentbased control for decentralised demand side management in the smart grid. In: Proceedings of the 10th international conference on autonomous agents and multiagent systems (AAMAS'11), vol 1, Taipei, 2-6 May 2011, pp 5-12

[23] Li N, Chen LJ, Low SH (2011) Optimal demand response based on utility maximization in power networks. In: Proceedings of the IEEE Power and Energy Society general meeting, San Diego, 24-29 July 2011, 8 pp

[24] Sortomme E, El-Sharkawi MA (2011) Optimal charging strategies for unidirectional vehicle-to-grid. IEEE Trans Smart Grid 2(1):131-138

[25] Cao YJ, Tang SW, Li CB et al (2012) An optimized EV charging model considering TOU price and SOC curve. IEEE Trans Smart Grid 3(1):388-393

[26] Shi WB, Wong VWS (2011) Real-time vehicle-to-grid control algorithm under price uncertainty. In: Proceedings of the 2011 IEEE international conference on smart grid communications, Brussels, 17-20 Oct 2011, pp 261-266

[27] Deilami S, Masoum AS, Moses PS et al (2011) Real-time coordination of plug-in electric vehicle charging in smart grids to minimize power losses and improve voltage profile. IEEE Trans Smart Grid 2(3):456-467

[28] White CD, Max Zhang K (2011) Using vehicle-to-grid technology for frequency regulation and peak-load reduction. J Power Sour 196(8):3972-3980

[29] Sundstrom O, Binding C (2012) Flexible charging optimization for electric vehicles considering distribution grid constraints. IEEE Trans Smart Grid 3(1):26-37

[30] O'Connell A, Flynn D, Richardson P et al (2012) Controlled charging of electric vehicles in residential distribution networks. In: Proceedings of the 3rd IEEE PES international conference and exhibition on innovative smart grid technologies (ISGT Europe'12), Berlin, 14-17 Oct 2012, 7 pp

[31] Acha S, Green TC, Shah N (2010) Effects of optimised plug-in hybrid vehicle charging strategies on electric distribution 
network losses. In: Proceedings of the IEEE PES transmission and distribution conference and exposition, New Orleans, 19-22 April 2010, 6 pp

[32] Wen CK, Chen JC, Teng JH et al (2012) Decentralized plug-in electric vehicle charging selection algorithm in power systems. IEEE Trans Smart Grid 3(4):1779-1789

[33] Stüdli S, Crisostomi E, Middleton R et al (2012) A flexible distributed framework for realising electric and plug-in hybrid vehicle charging policies. Int J Control 85(8):1130-1145

[34] Zhang P, Qian KJ, Zhou CK et al (2012) Demand response for optimisation of power systems demand due to EV charging load. In: Proceedings of the 2012 Asia-Pacific power and energy engineering conference (APPEEC'12), Shanghai, 27-29 Mar 2012, 4 pp

[35] Mets K, Verschueren T, Haerick W et al (2010) Optimizing smart energy control strategies for plug-in hybrid electric vehicle charging. In: Proceedings of the 2010 IEEE/IFIP network operations and management symposium workshops (NOMS Wksps'10), Osaka, 19-23 April 2010, pp 293-299

[36] Hu JJ, You S, Si CY et al (2013) Optimization and control methods for smart charging of electric vehicles facilitated by fleet operator: review and classification. Int J Distrib Energy Resour Smart Grids 10(1):383-397

[37] Ma ZJ, Callaway D, Hiskens I (2010) Decentralized charging control for large populations of plug-in electric vehicles: application of the Nash certainty equivalence principle. In: Proceedings of the IEEE international conference on control and applications (CCA'10), Yokohama, 8-10 Sept 2010, pp 191-195

[38] Ahn C, Li CT, Peng H (2011) Decentralized charging algorithm for electrified vehicles connected to smart grid. In: Proceedings of the 2011 American control conference (ACC'11), San Francisco, 29 June-1 July 2011, pp 3924-3929

[39] Li CT, Ahn C, Peng H et al (2012) Integration of plug-in electric vehicle charging and wind energy scheduling on electricity grid. In: Proceedings of the IEEE PES conference on innovative smart grid technologies (ISGT'12), Washington, DC, 16-20 Jan 2012, 7 pp

[40] Peças Lopes JA, Soares FJ, Almeida PM et al (2009) Smart charging strategies for electric vehicles: enhancing grid performance and maximizing the use of variable renewable energy resources. In: Proceedings of the 24th international battery, hybrid and fuel cell electric vehicle symposium and exhibition (EVS'09), Stavanger, 13-16 May 2009, 11 pp

[41] Aunedi M, Strbac G (2013) Efficient system integration of wind generation through smart charging of electric vehicles. In: Proceedings of the 8th international conference and exhibition on ecological vehicles and renewable energies (EVER'13), Monte Carlo, 27-30 March 2013, 12 pp

[42] Jin CR, Sheng X, Ghosh P (2013) Energy efficient algorithms for electric vehicle charging with intermittent renewable energy sources. In: Proceedings of the 2013 IEEE power and energy society general meeting, Vancouver, 21-15 July 2013, 6 pp

[43] Galus MD, Koch S, Andersson G (2011) Provision of load frequency control by PHEVs, controllable loads, and a cogeneration unit. IEEE Trans Ind Electron 58(10):4568-4582

[44] Wu CY, Mohsenian-Rad H, Huang JW (2012) Vehicle-to-aggregator interaction game. IEEE Trans Smart Grid 3(1):434-442

[45] Time-varying electricity price. Sustainable Energy Authority of Ireland (SEAI), Dublin

[46] Gan LW, Topcu U, Low S (2011) Optimal decentralized protocol for electric vehicle charging. In: Proceedings of the 50th IEEE conference on decision and control and European control conference (CDC-ECC'11), Orlando, 12-15 Dec 2011, pp 5798-5804

[47] Sundström O, Binding C (2010) Optimization methods to plan the charging of electric vehicle fleets. In: Proceedings of the international conference on control, communication and power engineering (CCPE'10), Chennai, 28 July 2010, pp 323-328

[48] Sortomme E, Hindi MM, MacPherson SDJ et al (2011) Coordinated charging of plug-in hybrid electric vehicles to minimize distribution system losses. IEEE Trans Smart Grid 2(1):198-205

[49] Shorten R, Wirth F, Leith D (2006) A positive systems model of TCP-like congestion control: Asymptotic results. IEEE/ACM Trans Netw 14(3):616-629

[50] Liu MM, McLoone S (2013) Enhanced AIMD-based decentralized residential charging of EVs. Trans Inst Meas Control. doi:10.1177/0142331213494100

[51] Phan DT, Xiong JJ, Ghosh S (2012) A distributed scheme for fair EV charging under transmission constraints. In: Proceedings of the 2012 American control conference (ACC'12), Montreal, 27-29 June 2012, pp 1053-1058

[52] Stüdli S, Crisostomi E, Middleton R (2014) Optimal real-time distributed V2G and G2V management of electric vehicles. Int J Control 87(6): 1153-1162

[53] Liu MM, McNamara P, McLoone S (2013) Fair charging strategies for EVs connected to a low-voltage distribution networks. In: Proceedings of the 4th IEEE PES conference on innovative smart grid technologies Europe (ISGT Europe'13), Lyngby, 6-9 Oct 2013, 6 pp

[54] OpenDSS: EPRI distribution system simulator. Electric Power Research Institute (EPRI), Palo Alto, 2012

[55] Electricity customer behaviour trial. Irish Social Science Data Archive (ISSDA). http://www.ucd.ie/issda/data/commissionfore nergyregulationcer/

[56] National Travel Survey (2009) Prn A11/1239, Central Statistics Office (CSO), Cork, 2011

Mingming LIU received his B.E. degree in Electronic Engineering (first class honours) from National University of Ireland Maynooth (now Maynooth University) in 2011. He is currently working towards a $\mathrm{PhD}$ degree in Applied Mathematics at the Hamilton Institute, Maynooth University. His current research interests include smart grid analysis, modelling and optimisation, nonlinear system dynamics and distributed control techniques.

Paul MCNAMARA graduated with a first class honours Bachelor of Electrical and Electronic Engineering degree in 2007 and a $\mathrm{PhD}$ in Centralised and Distributed Control of the Smart Grid in 2012, both from University College Cork, Ireland. Following his $\mathrm{PhD}$ he joined Maynooth University, Kildare, Ireland as postdoctoral researcher in Science Foundation Ireland funded Sustainable Electrical Energy Systems (SEES) research cluster. His research interests include centralised and distributed control, optimisation, and identification with applications to electrical power systems.

Robert SHORTEN graduated from University College Dublin (UCD) in 1990 with a First Class Honours B.E. degree in Electronic Engineering. He was awarded a PhD in 1996, also from UCD, while based in at Daimler-Benz Research in Berlin, Germany. From 1993 to 1996, Prof. Shorten was the holder of a Marie Curie Fellowship at Daimler-Benz Research to conduct research in the area of smart gearbox systems. Following a brief spell at the Center for Systems Science, Yale University, working with Professor K. S. Narendra, Prof. Shorten returned to Ireland as the holder of a European Presidency Fellowship in 1997. Prof. Shorten is a co-founder of the Hamilton Institute at NUI Maynooth, where he was a full Professor until March 2013. He was also a Visiting Professor at TU Berlin from 2011-2012. Professor Shorten is currently a senior research manager at IBM Research Ireland. Prof. Shorten's research spans a number of areas. He has been active in computer networking, automotive 
research, collaborative mobility (including smart transportation and electric vehicles), as well as basic control theory and linear algebra. His main field of theoretical research has been the study of hybrid dynamical systems.

Seán MCLOONE received an M.E. degree in Electrical and Electronic Engineering and a $\mathrm{PhD}$ in Control Engineering from Queen's University Belfast, Belfast, U.K. in 1992 and 1996, respectively. Following appointments as a Postdoctoral Research Fellow (1996-1997) and Lecturer at QUB (1998-2002) he joined the
Department of Electronic Engineering at the National University of Ireland Maynooth in 2002, where he served as Senior Lecturer (2005-2012) and Head of Department (2009-2012). He is currently a Professor and Director of the Energy Power and Intelligent Control (EPIC) Research Cluster at Queen's University Belfast. His research interests are in computational intelligence techniques and data analytics with application to modelling, optimisation and control of dynamical systems, advanced manufacturing, renewable energy and demand side management. 